\title{
Tregs: A Therapeutic Target for the Treatment of Portal Fibrosis?
}

\author{
Yuhua Xue $^{1} \cdot$ George Michalopoulos ${ }^{2}$
}

Received: 21 April 2015/Accepted: 23 April 2015/Published online: 14 May 2015

(C) Springer Science+Business Media New York 2015

Human science fragments everything in order to understand it. Kills everything in order to examine it.

—Leo Tolstoy

Primary cholangiopathies, diseases in which the biliary system is primarily affected, can be complicated by secondary fibrosis, with serious clinical consequences. Treatment of primary disorders is not available due to the lack of understanding of their etiology, such as for autoimmune disorders including primary biliary cirrhosis (PBC) and primary sclerosing cholangitis (PSC), or for structural defects such as biliary atresia, or for germline mutations as in the Alagille syndrome. Targeting the consequences of cholestasis, such as inflammation and fibrosis, creates treatment options, besides reducing the bile acid synthesis.

During cholestasis, activated myofibroblasts surrounding the expanded ductular area produce collagen and deposit other extracellular matrix proteins. Fundamental knowledge about how cholangiocytes expand, how expanded cholangiocytes initiate inflammation and guide the pattern of Th cells through Kupffer cells, and how Th17/ Treg balance affects the portal fibrosis is still lacking. This

Yuhua Xue

yux17@pitt.edu

George Michalopoulos

michalopoulosgk@upmc.edu

1 Department of Pathology, University of Pittsburgh, School of Medicine, 200 Lothrop St. BST-S453, Pittsburgh, PA 15261, USA

2 Department of Pathology, University of Pittsburgh, School of Medicine, 200 Lothrop St. BST-S410, Pittsburgh, PA 15261, USA editorial will discuss the available data regarding the etiopathogenesis of cholangiopathy-associated fibrosis, starting with a description of a commonly used animal models frequently employed in its study.

\section{Bile Duct Ligation (BDL) Model}

The BDL model has been widely used to study cholestatic liver injuries and fibrogenesis, even though its relation to clinical cholangiopathies has been questioned [1]. In response to experimental biliary obstruction, there is a substantial cholangiocyte proliferative response. Hepatic necrosis resulting from leakage of bile acids is followed by compensatory proliferation of cholangiocytes (Fig. 1), recruitment of inflammatory cells including lymphocytes, and activation of fibrogenic cells including portal fibroblasts, hepatic stellate cells (HSCs), and finally myofibroblasts. Generally, inflammatory cells, especially monocytes, will be recruited to the necrotic area and mediate secondary peri-necrotic fibrosis, whereas cholestasis-related fibrosis is predominantly periportal due to cholangiocyte, not monocyte activation.

\section{Tregs in the Liver}

CD4+ Th cells and other types of lymphocytes pass through liver every day. CD4+ Th cells can differentiate into four major subtypes [2], including Th1, Th2, Th17, and regulatory $\mathrm{T}$ cells (Tregs). Th1 lymphocytes assist $\mathrm{CD} 8+\mathrm{T}$ cells, Th2 lymphocytes mediate humoral immunity, and Th17 lymphocytes promote inflammation. Th17 and Tregs are antagonistically related. 


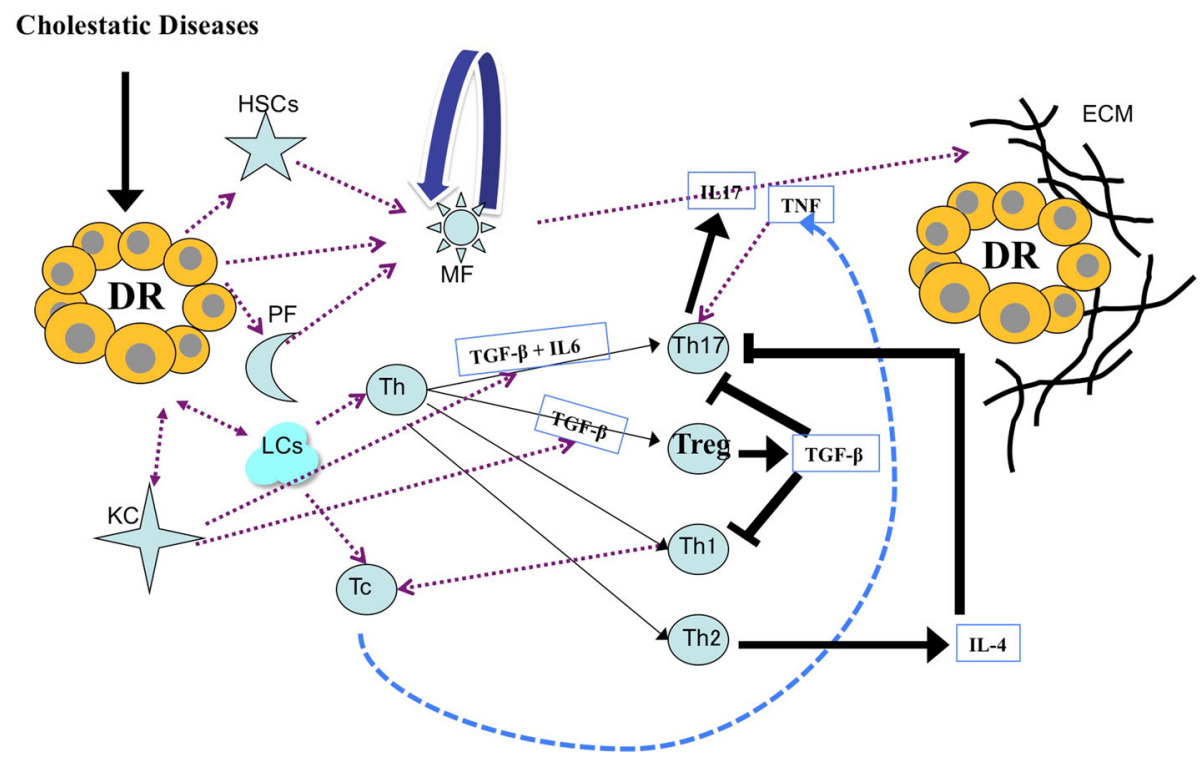

Fig. 1 The liver in chronic cholestatic injury: Key component of portal niche. Model highlighting key aspects of proposed contributions of hepatic cell types during portal fibrosis. In respond to cholestatic injuries, cholangiocytes proliferate to expand the ductular reaction (DR). Cholangiocytes recruit (red solid arrows) hepatic stellate cells (HSCs), myofibroblasts (MFs), portal fibroblasts (PFs), kupffer cells (KCs), and lymphocytes (LCs). Cholangiocytes, HSCs, PFs can transdifferentiate (red dashed arrows) into MFs. LCs differentiate (thick black solid arrows) into CD8+ T cytotoxic cell

\section{Cholangiocytes Dominate the Portal Niche in Th Differentiation}

The hepatic portal niche is defined as the unique microenvironment surrounding the portal tract, in which direct cell contact between parenchymal, mesenchymal, and immune cells, or indirect contact via autocrine and paracrine mediators is responsible for maintaining immune suppression under physiological conditions. Cholangiocytes in the niche are not just the physical barrier component of the bile transport system. In response to cholestatic injury, cholangiocytes proliferate and affect the cellular composition in the portal tract. Cholangiocytes attract lymphocytes to the portal niche and have potential to guide the Th cells differentiation pattern. Thus, the production of reactive cholangiocytes is considered to be one of the initial events of portal inflammation portal fibrosis [3]. Other cells types located within the portal niche include HSCs, Kupffer cells, and inflammatory cells, with the potential to signal to cholangiocytes via paracrine signaling to influence cholangiocyte behavior [4]. Although scientists are aware of the tight correlation between the intensity of cholestatic injury, cholangiocyte behavior, and portal fibrosis, at present it is not known how cholangiocyte expansion drives pre-myofibroblasts to differentiate into myofibroblasts, to become activated, and finally to initiate
(Tc) and CD4+ helper cell (Th). Th differentiate (thin black solid arrows) into different subtypes, including Th1, Th2, Th17 and Treg. Cytokines (blue dashed arrows) from KCs guide Th17/Treg balance, for example, TGF- $\beta$ promotes Treg differentiation, while in the presence of IL6, TGF- $\beta$ promotes Th17 but not Treg. IL17 and TNF from Th1/Tc axis promote extracellular matrix (ECM) production and deposition around expansion cholangiocytes/portal area. TGF- $\beta$ dependent TGF- $\beta$ production from Tregs negatively modulates the Th17 and Th1/Tc effects, thus decrease deposition ECM

ECM production (Fig. 1). Roh et al.'s study [5] has highlighted an important Th subtype in cholangiopathy-related portal niche changes, Tregs, which affect fibrosis progression directly or indirectly.

\section{Tregs, Myofibroblasts, Portal Niche, and Portal Fibrosis}

Myofibroblasts which distribute around the portal tract and initiate collagen deposition [6] are the main ECM-producing cells in cholestasis-induced portal fibrosis (Fig. 1). Tregs prevent autoimmune diseases such as PBC [7]. Although the exact functions of Tregs in the portal niche are still unknown, the model proposed from the work of Roh et al. [5] suggests that Tregs may indirectly modulate myofibroblasts by affecting Th17 and Th1/CD8+ Tc cytokine patterns, knowledge important for the development of future antifibrotic strategies, especially for those patients with deregulated Th17 and Th1 cytokine profiles. Currently, the mechanisms that convert quiescent HSCs into activated HSCs attract most attention. Studies investigating the transition from activated myofibroblasts to final ECM production enrich our understanding of the mechanism underlying portal fibrosis. Here, Roh et al. [5] have indicated an important subtype of CD4+ $\mathrm{T}$ lymphocytes, 
which modulate ECM production indirectly. Thus, it provides a promising and novel therapeutic strategy, which promises to modify the development of portal fibrosis regardless of primary liver insults.

\section{Methods to Assess Necrosis and Portal Fibrosis}

Several methods are commonly used in the analysis of liver parenchymal fibrosis such as assays of total tissue lysate, hydroxyproline, reverse transcription polymerase chain reaction (RT-PCR), or quantitative PCR or western blot analysis for collagen-related genes. Specific marker staining ( $\alpha$-smooth muscle antigen for myofibroblasts) and morphological analysis (Sirius red and Masson trichrome staining) are available for liver sections, used for the analysis of active and quiescent fibrosis, respectively, although in portal fibrosis, quantification or semi-quantification fibrosis is challenging. For example, analysis of total tissue lysates may be misleading, but analysis of specific portal tissue is technically challenging. Furthermore, the information obtained from the study of a few 2-D sections, either for specific marker staining or for morphological analysis, may grossly misrepresent the extent of in situ 3-D portal fibrosis. Roh et al. [5] analyzed scanned liver sections at high resolution, which with the addition of reconstruction software may have provided an accurate assessment of fibrosis in 3-D, while also analyzing total tissue lysate based at the transcriptional level.

\section{Perspective}

While Roh et al.'s paper in this issue describes the phenotype after Tregs depletion, some points need to be considered. BDL induces acute injury with fibrosis [8] around "big duct," which results from the extremely high physical pressure. This pattern is completely different from the chronic progressive partly compensatory "all size" duct fibrosis. Similarly, inflammatory reaction patterns might be different between these "acute" and "chronic" patterns. Thus, the pathogenesis of portal fibrosis is complicated, and cytokines involved in this process are cell-specific and context-dependent manner. For example, TGF- $\beta 1$ induces Tregs differentiation, while in the presence of IL6 results in
Th17 direction. In other settings, TGF- $\beta 1$ from hepatocytes and Kupffer cells promotes fibrosis. Thus, cytokine-based fibrosis therapy, although promising, is still in its infancy.

The discovery of Tregs expansion in the BDL model [9] by Roh et al. [5] has elegantly demonstrated the complex role of Tregs as potential antifibrotic agents. Tregs have potential as therapeutic cell targets for established inflammation or alternatively as guiding selective cytokine vector treatments for fibrosis immunotherapy. Studies of Tregs in vitro differentiation and culture [10] provide the possibility of Treg cell-based antifibrotic therapy that may include cell transplantation and dietary management.

\section{References}

1. Michalopoulos GK, Barua L, Bowen WC. Transdifferentiation of rat hepatocytes into biliary cells after bile duct ligation and toxic biliary injury. Hepatology. 2005;41:535-544.

2. Steinman L. A brief history of $\mathrm{T}(\mathrm{H}) 17$, the first major revision in the $\mathrm{T}(\mathrm{H}) 1 / \mathrm{T}(\mathrm{H}) 2$ hypothesis of $\mathrm{T}$ cell-mediated tissue damage. Nat Med. 2007;13:139-145.

3. Desmet VJ. Ludwig symposium on biliary disorders-part I. Pathogenesis of ductal plate abnormalities. Mayo Clin Proc. 1998;73:80-89.

4. Sanchez-Munoz D, Castellano-Megias VM, Romero-Gomez M. Expression of bcl-2 in ductular proliferation is related to periportal hepatic stellate cell activation and fibrosis progression in patients with autoimmune cholestasis. Dig Liver Dis. 2007;39:262-266.

5. Roh YS, Park S, Lim CW, Kim B. Depletion of Foxp3+ regulatory $\mathrm{T}$ cells promotes profibrogenic milieu of cholestasisinduced liver injury. Dig Dis Sci. (Epub ahead of print). doi:10. 1007/s10620-014-3438-2.

6. Bataller $\mathrm{R}$, Brenner DA. Liver fibrosis. J Clin Invest. 2005;115:209-218.

7. Lan RY, Cheng C, Lian ZX, et al. Liver-targeted and peripheral blood alterations of regulatory $\mathrm{T}$ cells in primary biliary cirrhosis. Hepatology. 2006;43:729-737.

8. Greenbaum LE, Wells RG. The role of stem cells in liver repair and fibrosis. Int J Biochem Cell Biol. 2011;43:222-229.

9. Katz SC, Ryan K, Ahmed N, et al. Obstructive jaundice expands intrahepatic regulatory $\mathrm{T}$ cells, which impair liver $\mathrm{T}$ lymphocyte function but modulate liver cholestasis and fibrosis. J Immunol. 2011;187:1150-1156.

10. Smits HH, Engering A, van der Kleij D, et al. Selective probiotic bacteria induce IL-10-producing regulatory $\mathrm{T}$ cells in vitro by modulating dendritic cell function through dendritic cell-specific intercellular adhesion molecule 3-grabbing nonintegrin. J Allergy Clin Immunol. 2005;115:1260-1267. 